\title{
Calibration of QUBIC: The Q \& U Bolometric Interferometer for Cosmology
}

\author{
James D. Murphy \\ National University of Ireland, Maynooth
}

\section{INTRODUCTION}

QUBIC (figure 1) is a ground based cosmology experiment aiming to detect and constrain the B-mode polarisation of the Cosmic Microwave Background. The $\Lambda$-CDM model (Big Bang theory) doesn't explain some current cosmological observations (Flatness problem/uniformity of the CMB). Inflation is proposed to explain the Flatness and uniformity of the CMB in the $\Lambda$-CDM model. It postulates that in the very early Universe, massive expansion of the Universe caused gravitational waves. This distortion in space-time would later (when photons decouple from matter) manifest itself as B-mode polarisation. QUBIC aims to find the faint B-mode polarisation in order to learn about the early evolution the Universe. QUBIC will begin observing in 2021 at Alto Chorillos near Salta, Argentina.

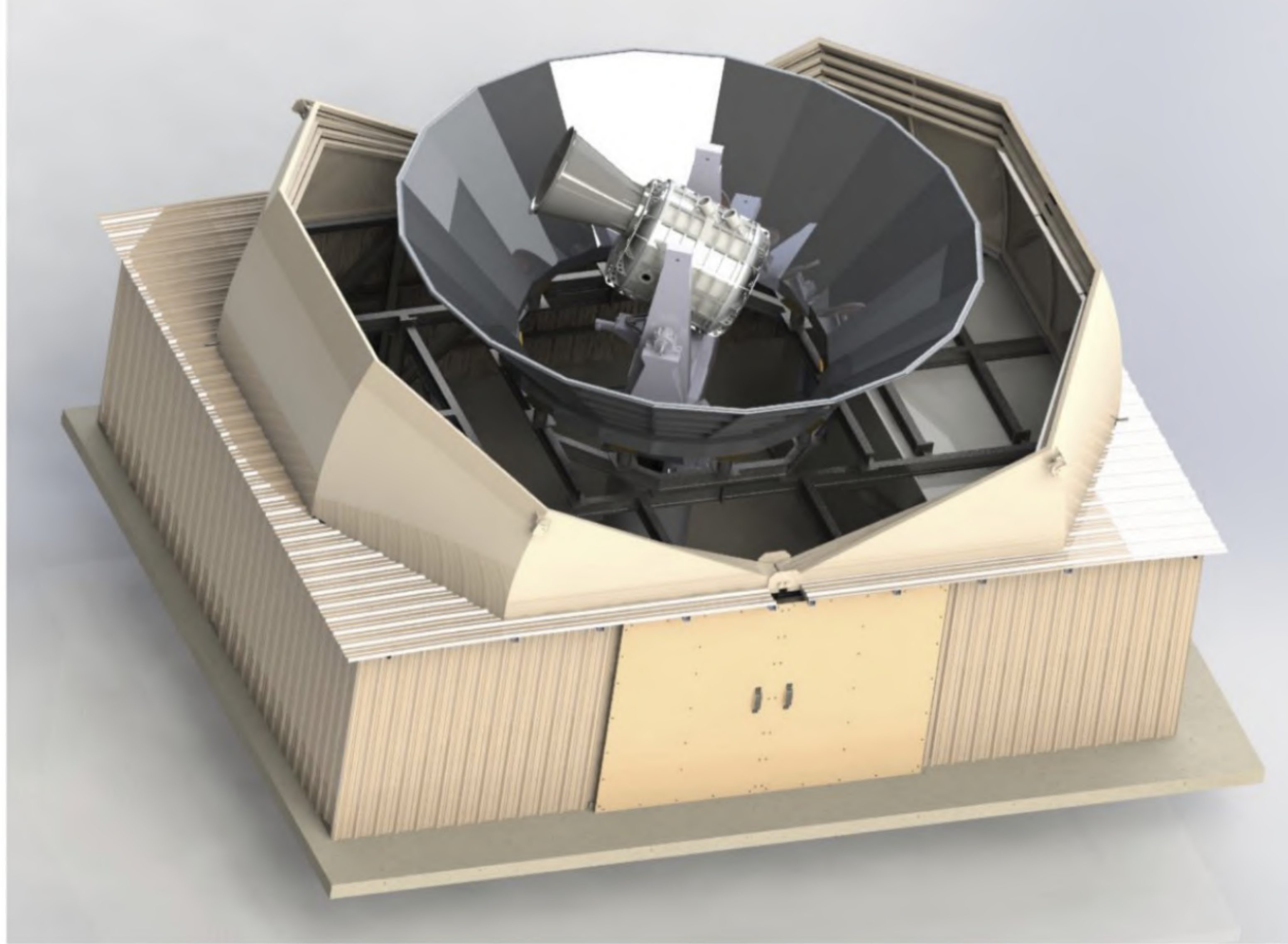

Figure 1. 3D render of QUBIC mounted in its dome on site at Alto Chorillos. QUBIC's mount, instrument, fore-baffle, ground-shield, and dome are shown.

QUBIC uses novel technologies (figure 2) necessary to filter out contributions from Galactic foregrounds, dust, and other galaxies. QUBIC will employ new techniques and technologies to find the B-mode signal such as:

\section{- Bolometric Interferometry}

- Horns at the aperture combine at the focal plane to form a synthetic image

\section{- Spectro-Imaging}

- $130-240 \mathrm{GHz}$ observed simultaneously to separate foregrounds

\section{Self-calibration}

- Switches in the horns enable excellent systematic control

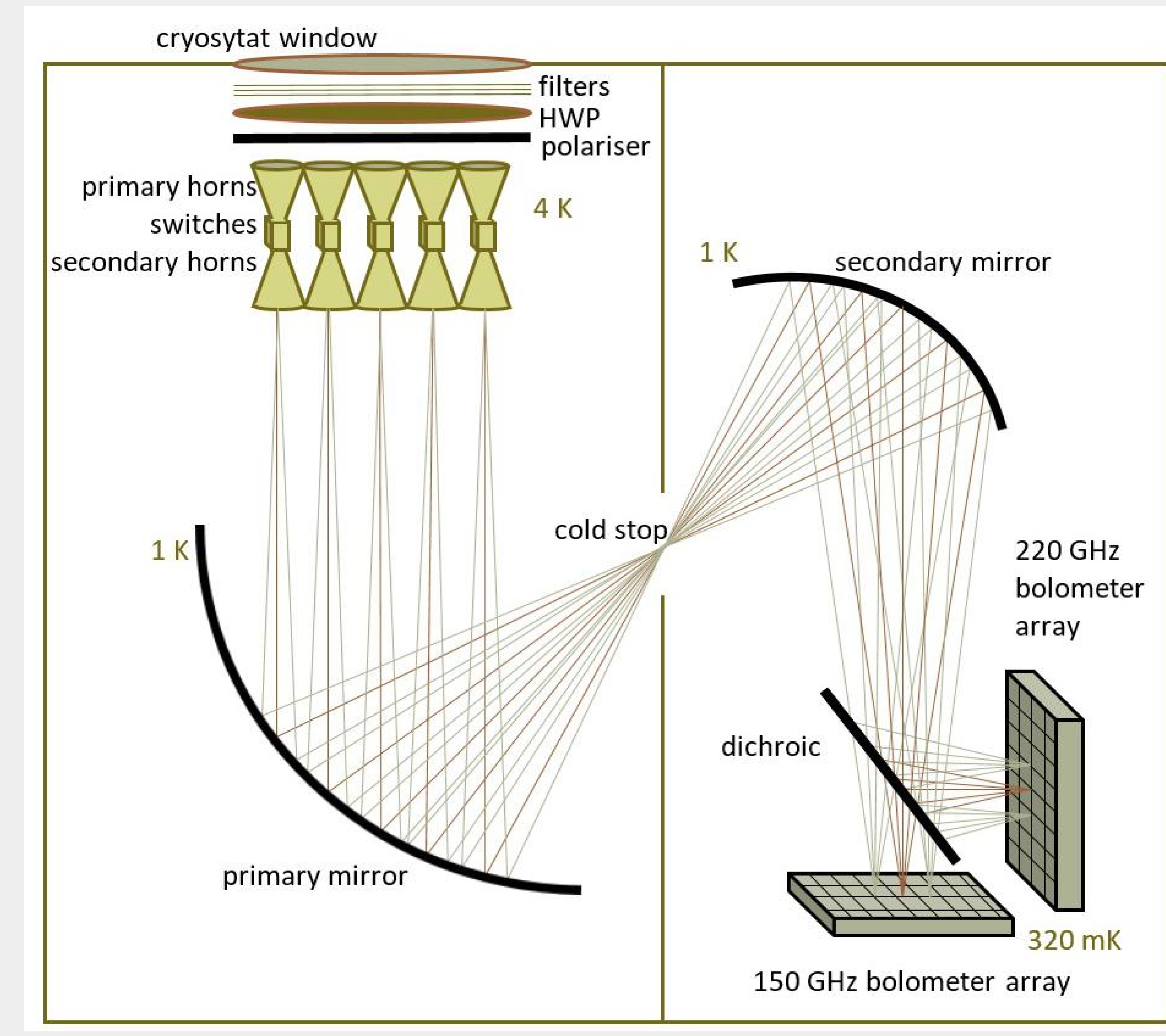

Figure 2. QUBIC's optical set-up inside the cryostats.

\section{Spectral Response}

- QUBIC's spectral response is tested and validated prior to observations (removing foregrounds)

- Results and methods from these tests form the ground-work for the end-to-end data processing pipeline

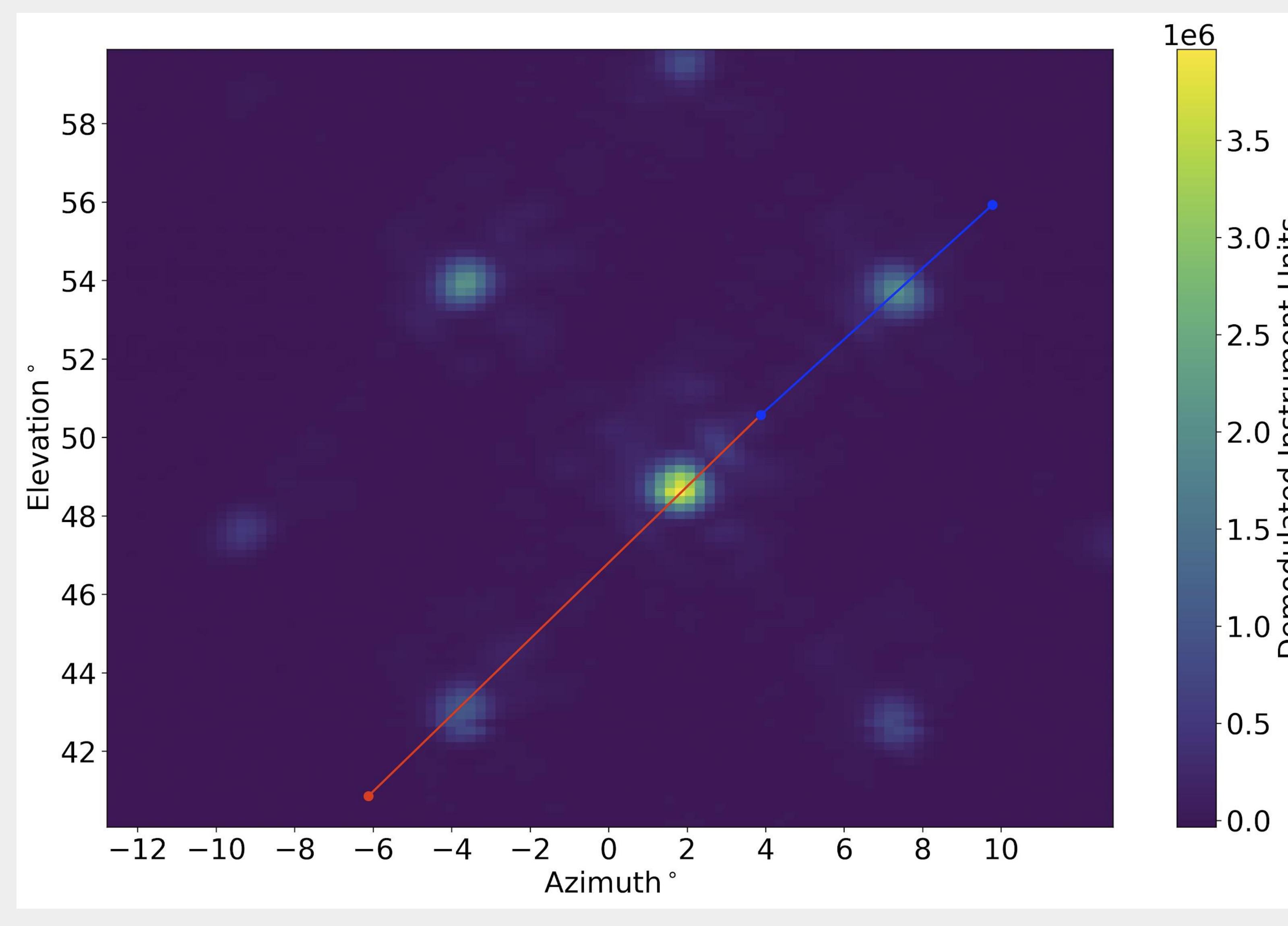

Figure 3. Demodulated interferometric pattern with cuts.

- Peak positions, FWHM, \& amplitude vary with frequency

- The laboratory test data is demodulated

- A demodulation optimisation is described in the paper

- Maps are made (figure 3) and measured (figure 4) for 130 , $150, \& 170 \mathrm{GHz}$ data-sets

- Measurements are compared to theoretical predictions and optical simulations (tables $1 \& 2$ )

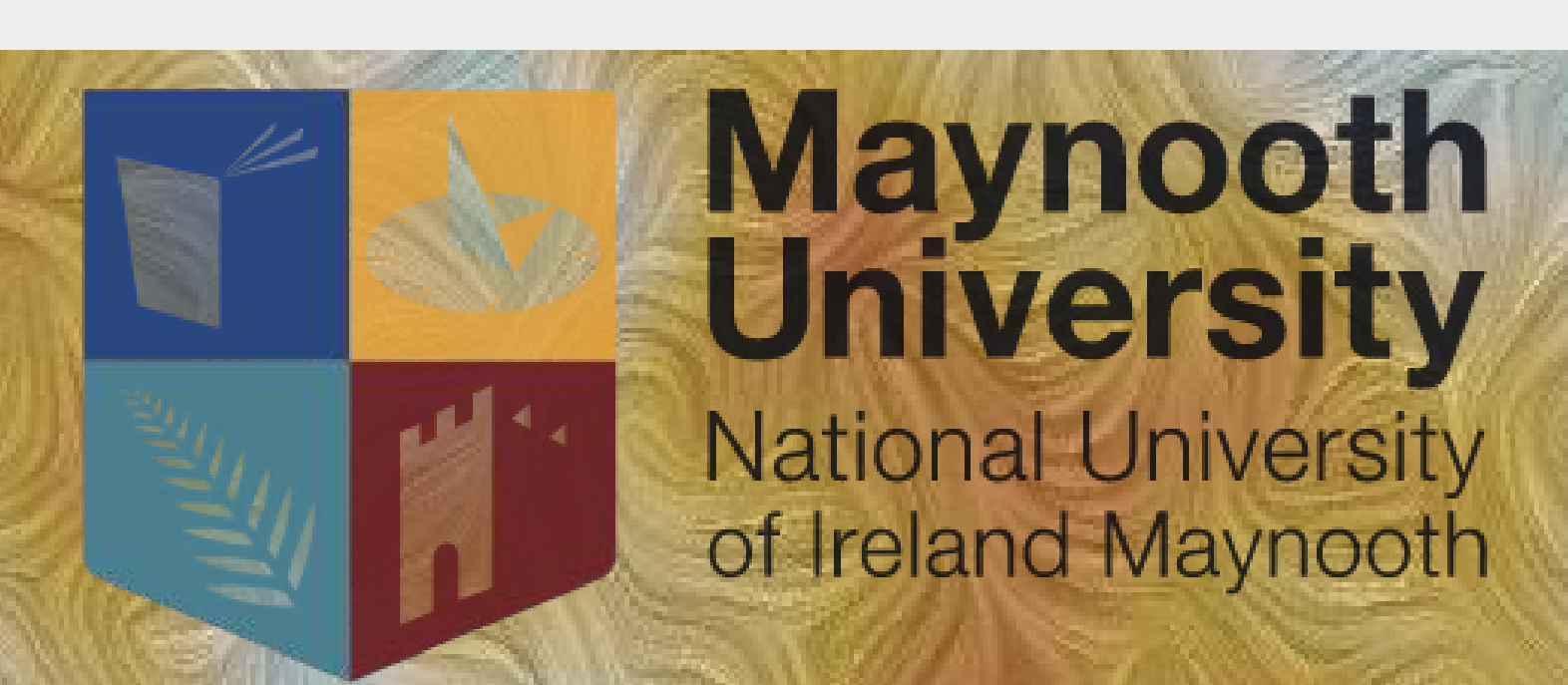

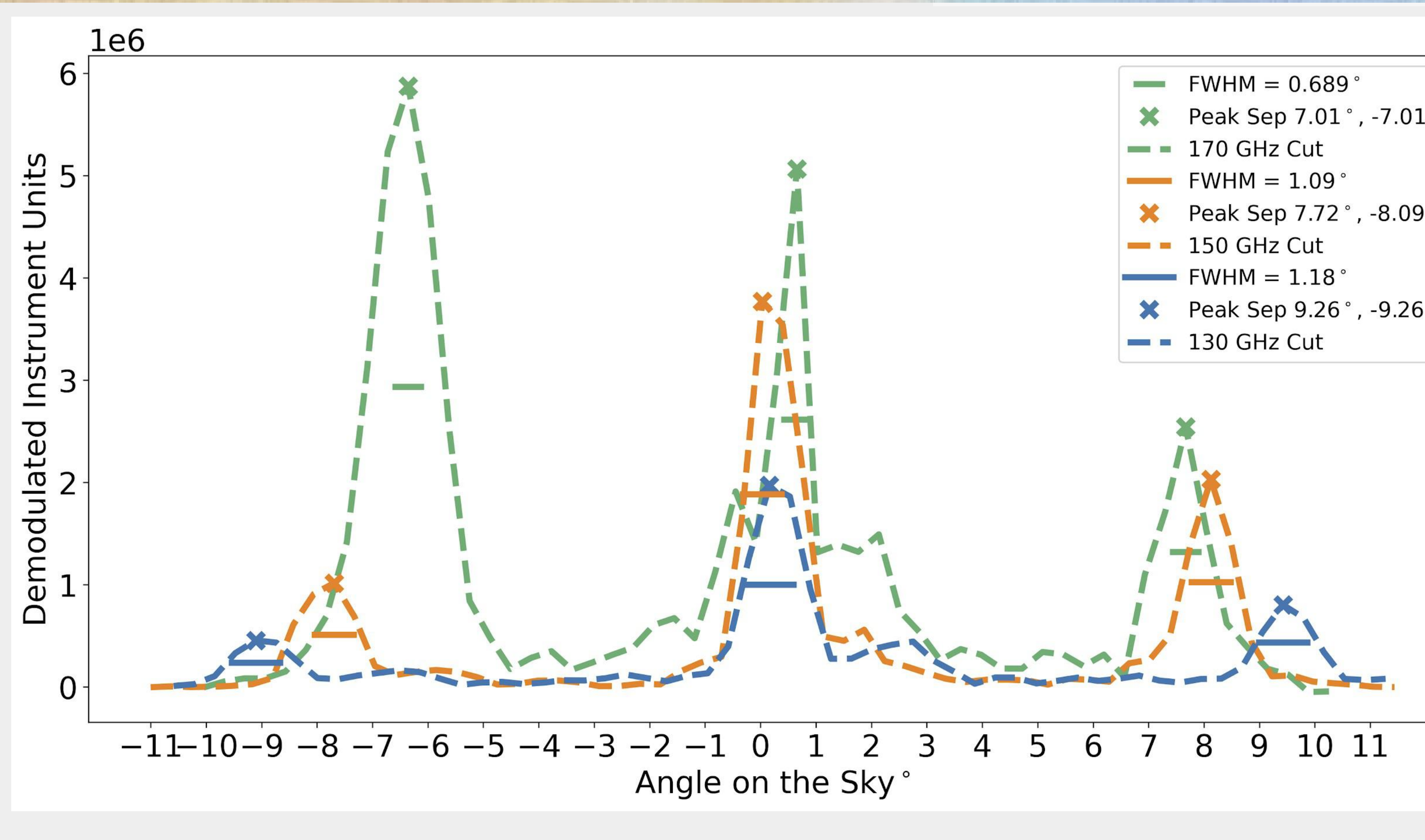

Figure 4. Cuts (for 130, 150, \& $170 \mathrm{GHz}$ ) from the maps (figure 3).

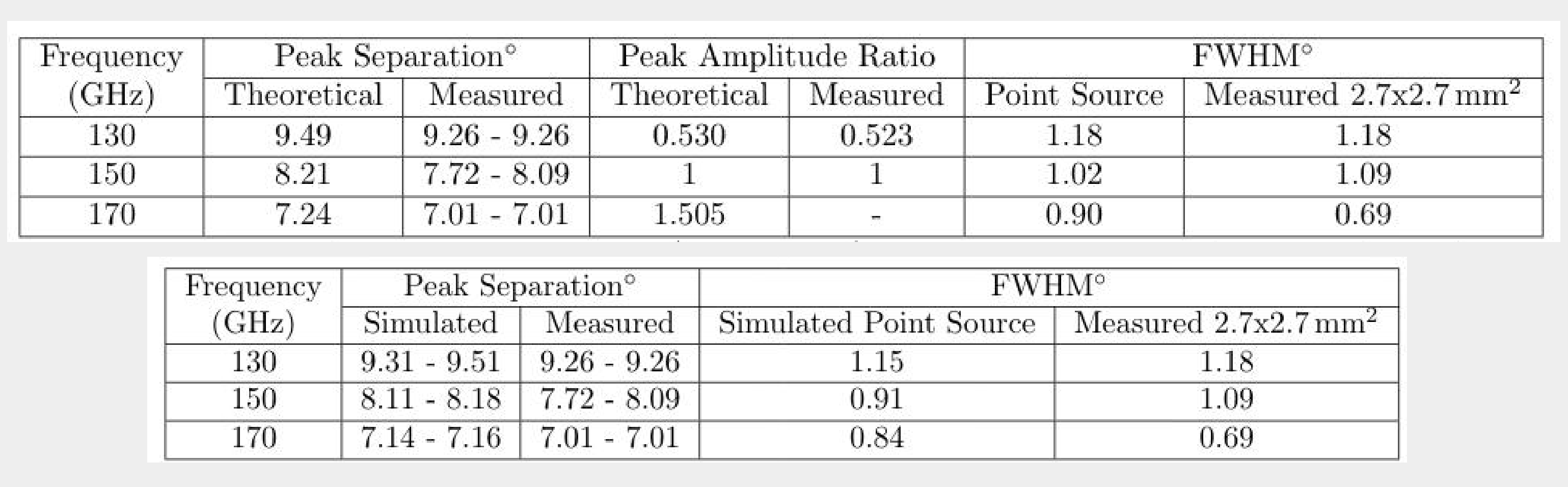

Tables 1 \& 2. These tables compare the measured instrument data with theoretical predictions and with optical simulations.

\section{Conclusions}

The spectral response of QUBIC was measured using laboratory test data. This was done by developing a method to optimise QUBIC's demodulation procedure, using the optimised demodulation parameters and making maps for $130,150, \& 170$ $\mathrm{GHz}$ data. The interferometric peak positions, FWHM, \& amplitudes were measured on the maps. The results were compared with theoretical predictions (ideal-imager) and with optical simulations (includes aberrations). Excellent agreement was found in both comparisons. However the $170 \mathrm{GHz}$ data saturated the detectors so the FWHM and amplitudes for this data are not suitable for comparison. These results show the QUBIC instrument is preforming well in terms of spectral response. The methods here will be developed and implemented into the end-to-end data processing pipeline.

\section{Acknowledgments}

J. D. Murphy and D. Burke acknowledge funding from the Irish Research Council under the Government of Ireland Postgraduate Scholarship Scheme. D. Gayer and S. Scully acknowledge funding from the National University of Ireland, Maynooth. D. Bennett acknowledges funding from Science Foundation Ireland. QUBIC is funded by the following agencies. France: ANR (Agence Nationale de la Recherche) 2012 and 2014, DIMACAV (Domaine d'Intérêt Majeur-Astronomie et Conditions d'Apparition de la Vie), CNRS/N2P3 (Centre national de la recherche scientifique/Institut national de physique nucléaire et de physique des particules), CNRS/NSU (Centre national de la recherche scientifique//nstitut national et al de sciences de l'univers). Italy: CNR/PNRA (Consiglio Nazionale delle Ricerche/Programma Nazionale Ricerche in Antartide) until 2016, INFN (Istituto Nazionale di Fisica Nucleare) since 2017. Argentina: MINCyT (Ministerio de Ciencia, Tecnología e Innovación), CNEA (Comisión Nacional de Energía Atómica), CONICET (Consejo Nacional de Investigaciones Cientifíicas y Técnicas). 